\title{
Vibrational Cooling Effect of Zeolite on Molecular Desorption Studied by Time-Resolved Laser Desorption Ionization Mass Spectrometry
}

\author{
Jiawei $\mathrm{Xu}^{1,2}$ and Tatsuya Fujino*1,2 \\ ${ }^{1}$ Bio-Nano Electronics Research Centre, Toyo University, Japan \\ ${ }^{2}$ Department of Applied Chemistry, Toyo University, Japan
}

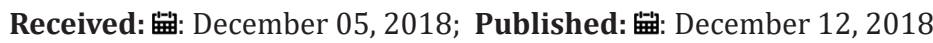

*Corresponding author: Tatsuya Fujino, Department of Applied Chemistry, Toyo University, Japan

\begin{abstract}
The desorption of the protonated species of 2,4,6-trihydroxyacetophenone (THAP) and 1'-hydroxy-2'-acetonaphthone (HAN) from their crystals was observed by using a time-resolved mass spectrometer and the desorption times were $7.2 \pm 2.3$ and $14.0 \pm 9.4 \mathrm{ps}$, respectively. The desorption time difference was explained by taking into consideration the number of intra- and intermolecular vibrational modes in THAP or HAN dimers. Then, the influence of the three-dimensional framework on the desorption process was investigated. The desorption time for THAP adsorbed on zeolite was $229.1 \pm 133 p s$. As the intermolecular vibrations between THAP and zeolite were directly connected to zeolite lattice vibrations with high density, desorption energy accumulation in those modes became difficult, resulting in the long desorption time. It was clarified that the three-dimensional framework suppressed molecular desorption because it acted as a heat bath, diffusing excess vibrational energy efficiently into its lattice vibrational modes.
\end{abstract}

Abbreviations: THAP: Trihydroxyacetophenone; LDI: Laser Desorption Ionization; MALDI MS: Matrix Assisted Laser Desorption Ionization Mass Spectrometry; HAN: Hydroxy Acetonaphthone

\section{Introduction}

Laser desorption ionization (LDI) mass spectrometry is one of the most reliable techniques for analyte detection. By using matrix molecules that absorb laser photons, "soft ionization," a process that does not decompose an analyte during ionization, becomes possible. This technique, called matrix-assisted laser desorption ionization mass spectrometry (MALDI MS), has been widely applied to the analysis of biopolymers, such as proteins or peptides, as its soft ionization potential enables observation of analyte-related ions with very few fragments [1-3]. However, MALDI MS has several drawbacks, including low ionization efficiency, protonated analyte peak suppression by alkali metal ion contaminants, and inapplicability to low molecular weight compounds due to the dissociation of matrix molecules. To overcome these drawbacks, many attempts have been made, including matrix-free techniques [4-7], application of nanometer-sized particles [8-10], and the use of a co-matrix with conventional organic matrix molecules [11-14]. Recently, we have revealed that zeolite is one of the most promising additives for MALDI MS. Zeolites are crystalline aluminosilicates with three-dimensional frameworks and nanometer-order cages. Zeolites have high catalytic activity due to the charge imbalance at the Si-O-Al bridging sites, and those sites are compensated typically by such cations as $\mathrm{H}^{+}$and $\mathrm{Na}^{+}$. Hydroxyl $(\mathrm{OH})$ groups having Brönsted acidity exist in $\mathrm{H}^{+}$-exchanged zeolite. It is known that the Brönsted acid site is responsible for the catalytic activity of zeolite. By using zeolites in MALDI MS, it was found that they prevented the dissociation of matrix molecules and enhanced the peak intensity of the protonated analyte [15].

The mechanism of the peak intensity enhancement could be understood as efficient proton supply from the Brönsted acid sites and stabilization of intra- and intermolecular proton transferred states by the strong polarity of zeolite [15]. However, the reason why zeolite prevents the dissociation of matrix molecules is still not clear, although it could be assumed that the excess vibrational energy of matrix molecules would be released to the 
lattice vibrations of zeolite. In this study, we examined how the three-dimensional framework as zeolites influenced molecular desorption by time-resolved mass spectrometry. Time-resolved mass spectrometry is a pump-probe type measurement [16-18]. By pumping, vibrational excitation of intermolecular dissociative modes takes place. Then, the molecule undergoes ionization including protonation and cation adduction by the probe, and the ion is released to the gas phase for detection. Therefore, the time required for molecular desorption can be detected by changing the delay time between pump and probe. It was understood that zeolite suppressed molecular desorption because it acted as a heat bath, diffusing excess vibrational energy efficiently into its lattice vibrational modes.

\section{Experimental}

2,4,6-Trihydroxyacetophenone (THAP) and 1'-hydroxy2'-acetonaphthone (HAN) were purchased from Sigma. $\mathrm{NH}_{4}^{+}$ terminated zeolite $\left(\mathrm{NH}_{4} \mathrm{M} 20\right)$ was supplied by the Catalysis Society of Japan. For time-resolved mass spectrometry, a Ti: sapphire laser with a regenerative amplifier (Spitfire, Spectra Physics) was used to provide femtosecond pulses $(800 \mathrm{~nm}, 0.8 \mathrm{~mJ}, 110 \mathrm{fs})$. The repetition rate was $10 \mathrm{~Hz}$. The output was frequency-doubled $(400 \mathrm{~nm})$ by an LBO crystal and then divided into pump and probe beams. The time delay between pump and probe was changed by the optical delay equipped in probe line. Mass spectrometry was performed under the condition that the spectrum could not be observed by single-beam excitation (pump or probe pulse only); pulse energy was $8 \mu \mathrm{J}$ for the pump and $10 \mu \mathrm{J}$ for the probe. Both pulses (pump and probe) were focused by a quartz lens and introduced into a laboratory-built time-of-flight (TOF) mass spectrometer. Solutions of THAP and HAN $\left(1.0 \times 10^{-2} \mathrm{~mol} \mathrm{dm}^{-3}\right)$ were prepared with a mixture of acetonitrile and water $(\mathrm{v} / \mathrm{v}=7: 3)$. Ten microliters of each solution were pipetted onto a stainless steel sample target and the solvent was allowed to evaporate. Thus-obtained crystals of THAP or HAN were subjected to time-resolved mass spectrometry. For the measurement of THAP on zeolite, THAP (16.8mg) was first mixed with NH4M20 (67.2mg) (weight ratio=1:4) in a mortar and pestle and the mixture was suspended in a mixture of acetonitrile and water $\left(\mathrm{v} / \mathrm{v}=7: 3,10 \mathrm{~mL} ; 1.0 \times 10^{-2} \mathrm{~mol} \mathrm{dm}^{-3}\right.$ for THAP). Then, ten microliters of the suspension was pipetted onto a stainless steel sample target. The obtained crystals of THAP $/ \mathrm{NH}_{4} \mathrm{M} 20$ were subjected to time-resolved mass spectrometry.

\section{Results and Discussion}

Figure 1a shows the mass spectrum of THAP crystals measured by pump pulse irradiation. No ions were detected by only the pump pulse irradiation. Figure $1 \mathrm{~b}$ shows the mass spectrum of THAP crystals measured by probe pulses. No ions were detected also by only the probe irradiation. In contrast, when the sample was irradiated by the pump and probe pulses at the same spot at a certain delay time, the situation became different. Figure 1c shows the mass spectrum of THAP crystals obtained by the pump-probe measurement (probing at 200ps delay after pumping). The peak of protonated THAP, $[\mathrm{THAP}+\mathrm{H}]^{+}$, was observed at $\mathrm{m} / \mathrm{z}=169$, whereas the peaks of $[\mathrm{THAP}+\mathrm{Na}]^{+}$and $[\mathrm{THAP}+\mathrm{K}]^{+}$were observed at $\mathrm{m} /$ $\mathrm{z}=191$ and 207, respectively. It is noted that this delay time was neither TOF nor the timing of ion extraction voltage $(10 \mathrm{~ms})$ after a laser shot, but the timing between the pump and probe pulses. In addition, any time resolution of electronic devices was not used in this study. Figure 1d shows the time dependence of the peak intensity of $[\mathrm{THAP}+\mathrm{H}]^{+}$. The peak intensity of $\left[\mathrm{THAP}+\mathrm{H}^{+}\right.$gradually increased with increasing delay time.

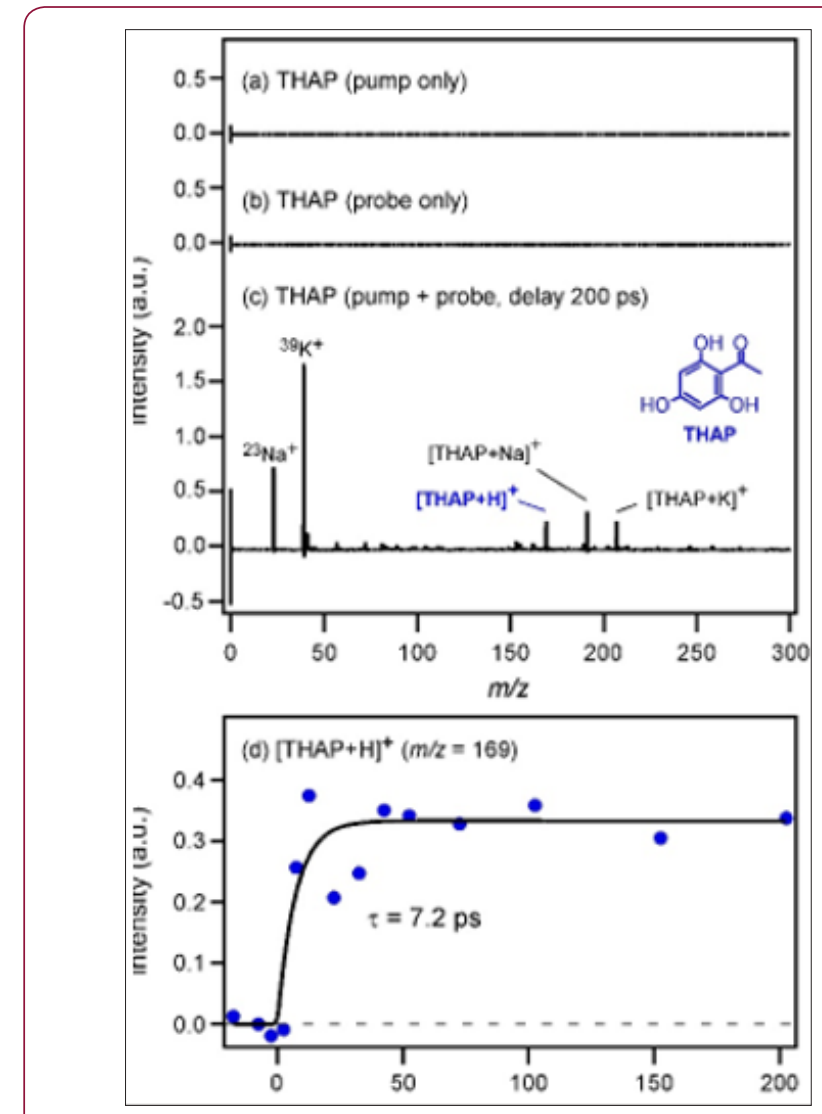

Figure 1: Mass spectrum of THAP crystals measured with

a) pump pulse

b) probe pulse

c) Time-resolved mass spectrum of THAP crystals measured with pump and probe irradiation (delay at 200ps)

d) Time dependence of peak intensity of $[\mathrm{THAP}+\mathrm{H}]^{+}$.

By fitting analysis using exponential functions, the time constant of the rise component was determined to be $7.2 \pm 2.3$ ps. Figure 2a shows the mass spectrum of HAN crystals measured with pump and probe irradiation (probing at 200ps delay after pumping). Peaks of $[\mathrm{HAN}]^{+}$and $[\mathrm{HAN}+\mathrm{H}]^{+}$were observed at $\mathrm{m} /$ $\mathrm{Z}=186$ and 187, respectively. The time dependence of the peak intensity of $[\mathrm{HAN}+\mathrm{H}]^{+}$is shown in Figure $2 \mathrm{~b}$. Although the $\mathrm{S} / \mathrm{N}$ ratio was not good, the rise time was determined by fitting analysis as 14.0 \pm 9.4 ps. In addition, the time dependence of the peak intensity of $[\mathrm{HAN}]^{+}$is shown in Figure 2c. Although $[\mathrm{HAN}]^{+}$and $[\mathrm{HAN}+\mathrm{H}]^{+}$ were different species, it was found that the intensity rise was also reproduced by the same exponential function having the lifetime of 14.0 ps. Therefore, it became clear that the rise component represented not the ionization efficiency but the desorption time, 
which was determined by the environment where each molecule existed. HAN has a similar molecular structure to THAP but a higher molecular weight. In addition, it was found that the absorbance of THAP at the excitation wavelength for time-resolved mass spectrometry $(400 \mathrm{~nm})$ was almost equal to that of HAN as shown in diffuse reflectance spectra of THAP and HAN crystals (Figure 3).
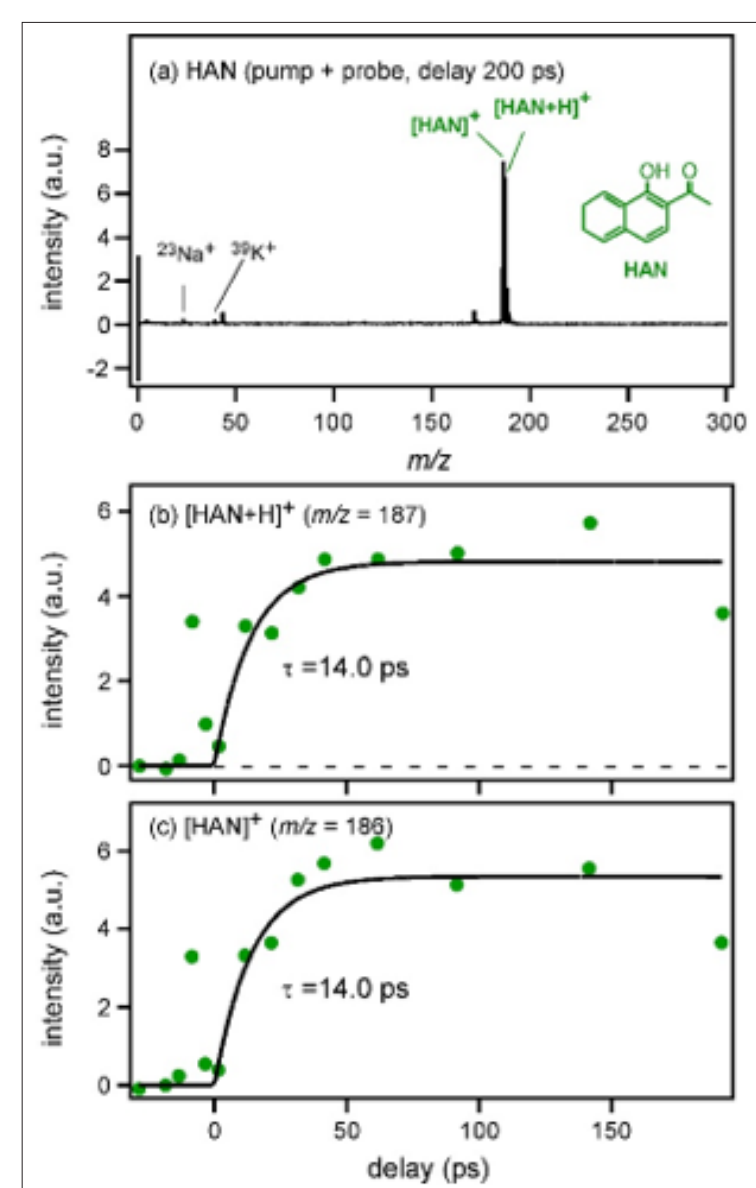

Figure 2: Time-resolved mass spectrum of HAN crystals measured with

a) pump and probe irradiation (delay at 200ps).

b) Time dependence of peak intensities of $[\mathrm{HAN}+\mathrm{H}]^{+}$

c) $[\mathrm{HAN}]^{+}$.

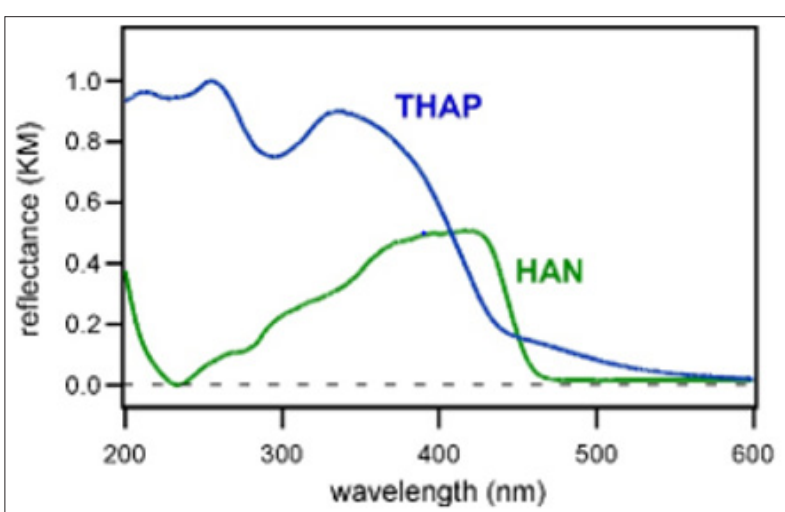

Figure 3: Diffuse reflectance spectra of THAP (blue) and HAN (green).
Therefore, the different appearance of the mass spectra is due to the difference in desorption and ionization properties of the two crystals. If we consider the $3 \mathrm{~N}-6$ rule for molecular vibrations ( $\mathrm{N}$ is the total number of atoms in the structure), the number of intramolecular vibrational modes of HAN would be larger than that of THAP. By pumping, THAP or HAN was photoexcited to the $S_{1}$ state. In the deactivation of the $S_{1}$ state to the $S_{0}$ state, photon energy was changed to intramolecular vibrational energy. After the intramolecular vibrational relaxation (IVR) process, those energies dissipated into the intermolecular vibrational modes. For the desorption of a molecule, multi-quantum excitation and breaking of every intermolecular vibrational mode are necessary. Therefore, it is possible that HAN requires much more time for IVR and vibrational energy dissipation into the intermolecular modes than THAP because the number of intramolecular vibrational modes of HAN is larger than that of THAP, leading to a longer desorption time. The desorption time difference between HAN and THAP could be explained also by taking those dimers into consideration. If a HAN dimer and the $3 \mathrm{~N}-6$ rule for the molecular vibrations are assumed, the calculated number of intermolecular vibrational modes would be 6 , which is equal to that of a THAP dimer.

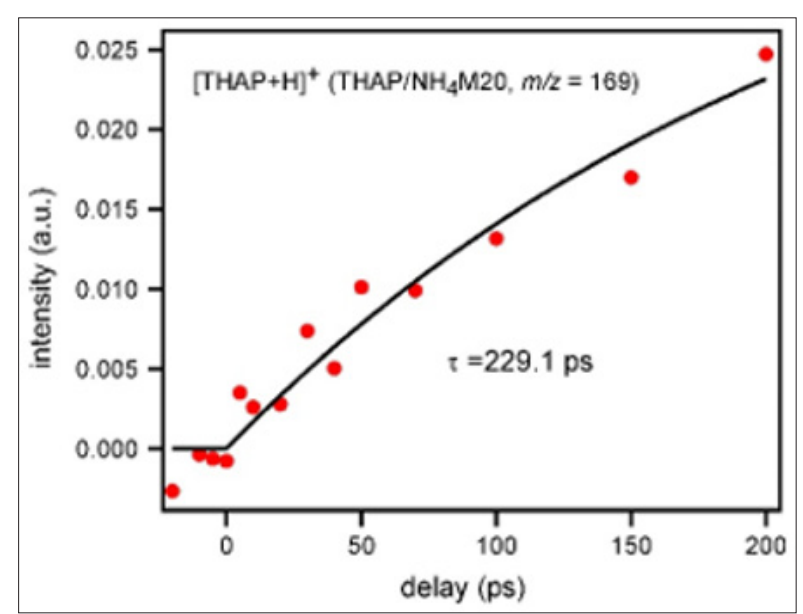

Figure 4: Time dependence of peak intensity of [THAP $+\mathrm{H}]^{+}$ from THAP/ $\mathrm{NH}_{4} \mathrm{M} 20$ measured by time-resolved mass spectrometry.

However, it is generally known that intermolecular vibrational modes often couple with intramolecular ones. Therefore, the number of intermolecular vibrational modes of HAN is larger than that of THAP practically because HAN has more intramolecular vibrational modes than THAP. The desorption time was determined by how rapid desorption-related intermolecular vibrational modes were excited. As HAN crystals as well as the dimer had more intermolecular vibrational modes than THAP crystals, it could be understood that much more time was necessary to excite those modes in HAN than THAP as the pump input energy $(400 \mathrm{~nm}$, $8 \mu \mathrm{J}$ ) was equal for both measurements. Then, how desorption proceeded when the molecules existed on a material with a high vibrational density of state was investigated. In this experiment, $\mathrm{NH}_{4}{ }^{+}$-terminated zeolite was used instead of $\mathrm{H}+$-type zeolite in order to extract the influence of only the three-dimensional framework 
on the desorption; ammonium ions blocked the active sites of zeolite, which led to minimum electrostatic attraction between the molecule and zeolite. (Figure 4) shows the time dependence of the peak intensity of $[\mathrm{THAP}+\mathrm{H}]^{+}$obtained from THAP/ $\mathrm{NH}_{4} \mathrm{M} 20$.

As the active sites of zeolite were blocked by ammonium ions and efficient proton supply to THAP was hindered, the peak intensity was almost one-tenth of that observed for THAP crystals (Figure 1c); only the effect of the three-dimensional framework on the desorption could be extracted. Although the $\mathrm{S} / \mathrm{N}$ ratio was not good, the time dependence of the peak intensity was fitted by exponential functions and the rise component was determined as $229.1 \pm 133 p$ s. For the desorption of THAP on zeolite surface, intermolecular vibrations between THAP and zeolite must be excited. However, as those vibrations were directly connected to zeolite lattice vibrations with high density, it became difficult to accumulate desorption energy in the intermolecular vibrational modes between THAP and zeolite. The rapid diffusion of vibrational energy by the zeolite lattice was responsible for the long desorption time (229.1ps) of THAP. Therefore, it was understood that zeolite offered such condition as diffusion of excess vibrational energy causing matrix as well as analyte fragmentation. Although molecular desorption became difficult by using zeolite, the peak intensity of analyte-related ions became strong when H+-type zeolite was used15 since ionization by efficient proton (cation) supply from Brönsted acid site was possible.

\section{Conclusion}

The desorption times of the protonated species of THAP $\left([\mathrm{THAP}+\mathrm{H}]^{+}\right)$and HAN $\left([\mathrm{HAN}+\mathrm{H}]^{+}\right)$from their crystals were determined to be $7.2 \pm 2.3$ and $14.0 \pm 9.4$ ps, respectively, with a pumpprobe type time-resolved mass spectrometer. This desorption time difference could be explained by considering the number of intermolecular vibrational modes in THAP or HAN dimers. It was clarified that a long time was required for the desorption of HAN, which had many intermolecular vibrational modes. Then, the influence of the three-dimensional framework on the desorption process was investigated. The desorption time for THAP adsorbed on zeolite was $229.1 \pm 133$ ps. For the desorption of THAP on zeolite surface, the intermolecular vibrations between THAP and zeolite must be excited. As the intermolecular vibrations between THAP and zeolite were directly connected to zeolite lattice vibrations with high density, it became clear that desorption energy accumulation in those modes was difficult, leading to the long desorption time.

\section{References}

1. K Tanaka, H Waki, Y Ido, S Akita, Y Yoshida (1988) Protein and polymer analyses up to m/z 100000 by laser ionization time of flight mass spectrometry 2: 151 .

2. F Hillenkamp, M Karas, RC Beavis, BT Chait (1991) Matrix-Assisted Laser Desorption/Ionization Mass Spectrometry of Biopolymers. Analytical chemistry 63(24): 1193A-1203A.
3. F Hillenkamp, M Karas(2000) Matrix-assisted laser desorption ionisation an experience. International Journal of Mass Spectrometry 200(1-3): 71-77.

4. EP Go, JV Apon, G Luo, A Saghatelian, RH Daniels, et al. (2005) Desorption/ionization on silicon nanowires 77(6): 1641-1646.

5. SA Trauger, EP Go, Z Shen, JV Apon, BJ Compton, et al. (2004) High sensitivity and analyte capture with desorption/ionization mass spectrometry on silylated porous silicon. Anal Chem76(15): 4484-4489.

6. J Wei, J M Buriak, G Siuzdak (1999) Desorption ionization mass spectrometry on porous silicon. Nature 399(6733): 243-246.

7. TR Northen, O Yanes, MT Northen, D Marrinucci, W Uritboonthai, et al. (2007) Clathrate nanostructures for mass spectrometry. Nature 449(7165): 1033-1036.

8. YF Huang, HT Chang (2008) Cancer Cell Targeting Using Multiple Aptamers Conjugated on Nanorods. Anal Chem 80(3): 567-572.

9. H Kawasaki, T Yonezawa, T Watanabe, R Arakawa (2007) Platinum Nanoflowers for Surface-Assisted Laser Desorption/Ionization Mass Spectrometry of Biomolecules. J Phys Chem C 111(44): 16278-16283.

10.X Wen, S Dagan, VH Wysocki (2007) Small-Molecule Analysis with Silicon-Nanoparticle-Assisted Laser Desorption/Ionization Mass Spectrometry. Anal Chem 79(2): 434-444.

11. S Kjellstro, ON Jensen (2004) Phosphoric Acid as a Matrix Additive for MALDI MS Analysis of Phosphopeptides and Phosphoproteins. Anal Chem $76:$ 5109-5117.

12. T Nishikaze, M Takayama (2007) Disappearance of interfering alkalimetal adducted peaks from matrix-assisted laser desorption/ionization mass spectra of peptides with serine addition to alpha-cyano-4hydroxycinnamic acid matrix. Rapid Commun Mass Spectrom 21(20): 3345-3351.

13. C Koster, JA Castro, CL Wilkins (1992) High-Resolution Matrix-Assisted Laser Desorption/Ionization of Biomolecules by Fourier Transform Mass Spectrometry. J Am Chem Soc 114 : 7572-7574.

14. T Kobayashi, H Kawai, T Suzuki, T Kawanishi, T Hayakawa (2004) Improved sensitivity for insulin in matrix-assisted laser desorption/ ionization time-of-flight mass spectrometry by premixing alpha-cyano4-hydroxycinnamic acid matrix with transferrin. Rapid Commun Mass Spectrom 18(10): 1156-1160.

15. Y Komori, H Shima, T Fujino, JN Kondo, K. Hashimoto et al. (2010) Pronounced Selectivity in Matrix-Assisted Laser Desorption-Ionization Mass Spectrometry with 2,4,6-Trihydroxyacetophenone on a Zeolite Surface: Intensity Enhancement of Protonated Peptides and Suppression of Matrix-Related Ions. J Phys Chem C 114 : 1593-1600.

16. R Knochenmuss, A Vertes (2000) Time-delayed 2-Pulse Studies of MALDI Matrix Ionization Mechanisms. J Phys Chem 104(23): 5406-5410.

17. Y Minegishi, D Morimoto, J Matsumoto, H Shiromaru, K Hashimoto et al. (2012) Desorption Dynamics of Tetracene Ion from Tetracene-Doped Anthracene Crystals Studied by Femtosecond Time-Resolved Mass Spectrometry. J Phys Chem C 116(4): 3059-3064.

18. Y Minegishi, J Matsumoto, H Shiromaru, K Hashimoto, T Fujino (2013) Femtosecond time-resolved laser desorption/ionization mass spectrometry of perylene crystals using Raman-induced vibrational excitation. Chem Phys Lett 584: 14-17. 


\section{ISSN: 2574-1241}

DOI: 10.26717/BJSTR.2018.12.002191

Tatsuya Fujino. Biomed J Sci \& Tech Res

(C) (P) This work is licensed under Creative

Submission Link: https://biomedres.us/submit-manuscript.php

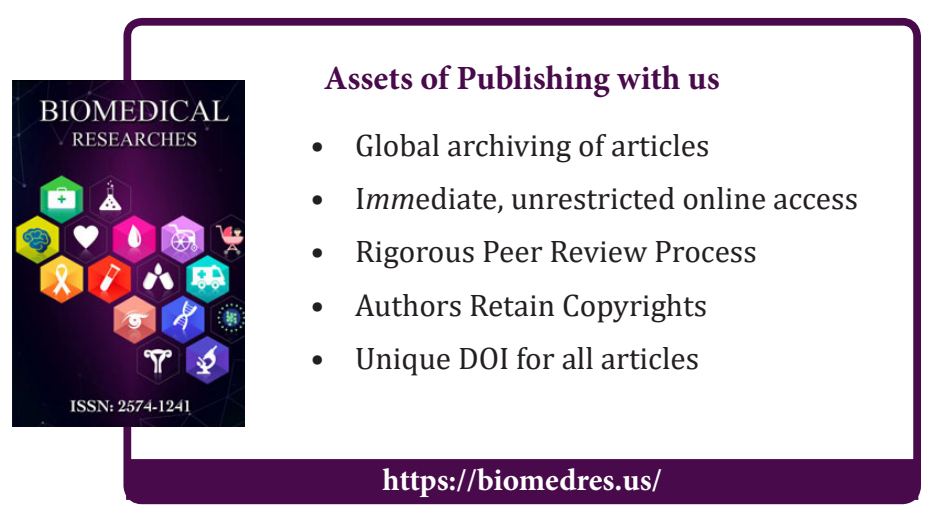

